\title{
Defect-mediated Rashba engineering for optimizing electrical transport in thermoelectric BiTeI
}

\author{
Xin $\mathrm{Li}^{1}$, Ye Sheng ${ }^{1}$, Lihua Wu $\mathbb{C}^{2}$, Shunbo $\mathrm{Hu}^{1}$, Jiong Yang $\mathbb{\mathbb { C }}^{1 凶}$, David J. Singh $\mathbb{C}^{3}$, Jihui Yang $\mathbb{C}^{2}$ and Wenqing Zhang $\mathbb{\mathbb { D }}^{4,5 凶}$
}

The Rashba effect plays a vital role in electronic structures and related functional properties. The strength of the Rashba effect can be measured by the Rashba parameter $a_{R}$; it is desirable to manipulate $a_{R}$ to control the functional properties. The current work illustrates how $a_{R}$ can be systematically tuned by doping, taking BiTel as an example. A five-point-spin-texture method is proposed to efficiently screen doped BiTel systems with the Rashba effect. Our results show that $a_{\mathrm{R}}$ in doped BiTel can be manipulated within the range of $0-4.05 \mathrm{eV} \AA$ by doping different elements. The dopants change $a_{\mathrm{R}}$ by affecting both the spin-orbit coupling strength and band gap. Some dopants with low atomic masses give rise to unexpected and sizable $a_{R}$, mainly due to the local strains. The calculated electrical transport properties reveal an optimal $a_{R}$ range of $2.75-3.55 \mathrm{eV} \AA$ for maximizing the thermoelectric power factors. $a_{R}$ thus serves as an effective indicator for high-throughput screening of proper dopants and subsequently reveals a few promising Rashba thermoelectrics. This work demonstrates the feasibility of defect-mediated Rashba engineering for optimizing the thermoelectric properties, as well as for manipulating other spin-related functional properties.

npj Computational Materials (2020)6:107; https://doi.org/10.1038/s41524-020-00378-4

\section{INTRODUCTION}

The Rashba effect plays a critical role in controlling the electronic structure and therefore the functional properties of semiconductors, including topological insulators and thermoelectrics ${ }^{1-7}$. The Bychkov-Rashba form of the spin-orbit coupling (SOC) perturbation Hamiltonian, $\mathbf{H}_{\mathrm{R}}=a_{\mathrm{R}}(\boldsymbol{\sigma} \times \mathbf{k}) \cdot \mathbf{z}^{8}$, is used to describe the strength of the Rashba effect, where $a_{\mathrm{R}}$ is the Rashba parameter $\left(a_{\mathrm{R}} \propto E_{\mathbf{z}}, E_{\mathbf{z}}\right.$ is the electric field $\left.{ }^{9}\right), \boldsymbol{\sigma}$ the Pauli spin matrices, $\mathbf{k}$ the momentum, and $\mathbf{z}$ the direction of the electric field. Systems with a strong Rashba effect generally have a large Rashba parameter $a_{\mathrm{R}}=2 E_{0} /\left|\mathbf{k}_{\mathbf{0}}\right|$ (Fig. 1a), where $E_{0}$ is the Rashba energy and $\mathbf{k}_{\mathbf{0}}$ is the momentum shift. The band parameter $a_{R}$ is thus an intrinsic measure of the Rashba strength in materials.

Rashba materials are generally noncentrosymmetric bulk materials or two-dimensional materials, such as semiconductor heterostructures or quantum wells with symmetry breaking arising from external electric fields or other gradients ${ }^{10-14}$. Strong Rashba effects are also found in several bulk materials, such as $\operatorname{BiTeX}(\mathrm{X}=\mathrm{I}, \mathrm{Br}, \mathrm{Cl})^{9,15-18}, a$-IVATe (IVA $\left.=\mathrm{Ge}, \mathrm{Sn}\right)^{1,19}$, and perovskites $^{20,21}$. Identifying new materials with giant $a_{\mathrm{R}}$, especially singlephase bulk materials, is desirable. Furthermore, optimizing $a_{\mathrm{R}}$ based on existing Rashba systems is also of considerable interest. This can be done by applying an external electric field ${ }^{22}$, polarization ${ }^{1}$, or strain ${ }^{11}$. Importantly, because the Rashba effect changes the electronic structures, there can be strong effects on the electrical transport properties of thermoelectrics. This can occur through modification of the density of states (DOS) ${ }^{23}$ and presumably also through changes in dispersion relations, both of which are important for the electrical resistivity.

A high thermoelectric figure of merit, $Z T=S^{2} T / k \rho$, is of vital importance for thermoelectrics. Here, $S$ is the thermopower, $\rho$ the electrical resistivity, $T$ the absolute temperature, and $\kappa$ the total thermal conductivity. Enhancing the power factor $\left(P F=S^{2} / \rho\right)$ and reducing the thermal conductivity of materials are two strategies to improve $Z T$. For electrical transport, the thermopower, in the numerator, and the electrical resistivity, in the denominator, are both positively correlated with the DOS. This reflects the fact that in a parabolic band model, a higher effective mass leads to enhanced $S$. It also leads to reduced mobility, meaning enhanced resistivity and lowered conductivity. This competition between the thermopower and electrical resistivity in optimizing PF may cause $\mathrm{PF}$ to be extremely sensitive to band features, such as the Rashba parameter $a_{R}$. Thus, it is desirable to directly tune $a_{R}$ to optimize the PFs of Rashba thermoelectrics. The resulting possibility of Rashba engineering offers another degree of freedom for tuning the electrical transport properties beyond traditional band and scattering engineerings in the optimization of thermoelectrics ${ }^{24}$. The ability to manipulate $a_{\mathrm{R}}$ may also have important consequences for other areas of semiconductor physics for which the Rashba effect plays an important role. However, the rational control of $a_{\mathrm{R}}$ is highly nontrivial because this parameter depends not only on the strength of the SOC but also on the interplay of the SOC and the strength of centrosymmetry breaking, as manifested in the particular electronic states in question.

Here, we demonstrate the control of $a_{R}$ in BiTel over a wide range of $0-4.05 \mathrm{eV} \AA$. This is realized by elemental doping on different atomic sites in the compound. We have developed a fivepoint-spin-texture (FPST) method to screen 32 doped BiTel compositions out of 62 candidates. This method provides an efficient approach for identifying new Rashba materials in a highthroughput (HTP) manner. By calculating the electronic structures for the 32 doped BiTel compositions with the Rashba effect, a dopant $-a_{\mathrm{R}}$ map is constructed. Furthermore, the relationship between $a_{\mathrm{R}}$ and PF is elucidated for these materials. This leads

\footnotetext{
${ }^{1}$ Materials Genome Institute, Shanghai University, 99 Shangda Road, 200444 Shanghai, China. ${ }^{2}$ Materials Science and Engineering Department, University of Washington, Seattle, WA 98195, USA. ${ }^{3}$ Department of Physics and Astronomy and Department of Chemistry, University of Missouri, Columbia, MO 65211, USA. ${ }^{4}$ Department of Physics and Shenzhen Institute for Quantum Science \& Technology, Southern University of Science and Technology, 518055 Shenzhen, Guangdong, China. ${ }^{5}$ Guangdong Provincial Key Laboratory of Computational Science and Material Design, and Shenzhen Key Laboratory of Advanced Quantum Functional Materials and Devices, Southern University of Science and Technology, 518055 Shenzhen, Guangdong, China. ${ }^{凶}$ email: jiongy@t.shu.edu.cn; zhangwq@sustech.edu.cn
} 
remarkably to an optimal range of $a_{R}$ for maximizing PF. Based on these results and the mapping between the composition and $a_{R}$ a few promising Rashba thermoelectrics are proposed. These may be useful in guiding experimental work.

\section{RESULTS AND DISCUSSION}

The dopant $-a_{R}$ map for BiTel

BiTel occurs in the space group $P 3 m 1$, which has no inversion symmetry. The strong chemical difference between Te and I enhances the effect of inversion symmetry breaking and the spin-orbit influence on the electronic structure. This is combined with the strong SOC in BiTel due to the heavy masses and $p$-electron nature of the constituent elements. Manipulating the Rashba effect then depends on manipulating this chemistry. The original degenerate band structure without SOC (Fig. 1a dotted line) of BiTel is thus subject to sizable Rashba spin splitting, characterized by $a_{\mathrm{R}}=2 E_{0} /\left|\mathbf{k}_{\mathbf{0}}\right|$ (Fig. 1a solid line), at both the conduction band minimum (CBM) and the valence band maximum (VBM) (Supplementary Fig. 1b), centered at the $A$ point, as shown. Here, we demonstrate how elemental doping regulates the $a_{\mathrm{R}}$ of BiTel; 62 elemental dopants with a doping content of 0.11 are tested. The atomic sites of the dopants are shown in Fig. 1c (see the "Methods" section for details). To quickly screen systems with the Rashba effect, we propose a FPST (discussed later) method and implement it to study the systems discussed. This method identifies 33 systems. Band structures for the 33 systems are then calculated, and 32 of them converge with finite $a_{\mathrm{R}} \mathrm{s}$. All $32 a_{\mathrm{R}} \mathrm{s}$ are shown in Fig. 1d, classified by the groups of the dopants in the periodic table (the VIII group is divided into the Fe group, Co group, and $\mathrm{Ni}$ group). The hollow diamond indicates the $a_{\mathrm{R}}$ of pristine BiTel $\left(a_{\mathrm{R}}=3.71 \mathrm{eV} \AA\right)$.

\section{The FPST method}

Figure $2 a-d$ shows the carrier pocket spin texture of BiTel. The energy contours are centered at the A point, with the energy level labeled by the gray dashed line in the inset of Fig. 2e (below $E_{0}$ ). The color strength denotes the spin polarization intensity along the different directions. As shown in Fig. 2d, the Rashba-induced spin component is perpendicular to the $z$ direction, i.e., $S_{z} \approx 0$. In the $x y$ plane (Fig. $2 a-c), S_{x}(\mathbf{k})=-S_{x}(-\mathbf{k})$ and $S_{y}(\mathbf{k})=-S_{y}(-\mathbf{k})$. Therefore, the spin components of $k$-point pairs around the $A$ point (or other Rashba degeneracy points in other materials) can be used to quickly screen the doped BiTel for the Rashba effect strength.

To take advantage of this, we propose the FPST method, which is based on the characteristics of the spin texture. We have developed and applied this FPST approach for doped BiTel, as shown in Fig. 2e. Specifically, the steps are as follows: (1) calculate the energy eigenvalues and spin components of five $\mathbf{k}$-points ( $\mathbf{k}_{c}$ $\mathbf{k}_{1}, \mathbf{k}_{2}, \mathbf{k}_{3}, \mathbf{k}_{4}$ ) for each system. $\mathbf{k}_{c}$ is the A point in the case of BiTel, and $\mathbf{k}_{1}, \mathbf{k}_{2}, \mathbf{k}_{3}$, and $\mathbf{k}_{4}$ are two $\mathbf{k}$ pairs $\left(\mathbf{k}_{1}=-\mathbf{k}_{2}, \mathbf{k}_{3}=-\mathbf{k}_{4}\right)$ close to $\mathbf{k}_{c}$. (2) Find two degenerate bands at the A point $\left(\mathbf{k}_{c}\right)$ around the Fermi level $E_{\mathrm{F}}$. Here, we scan the five closest bands with respect to $E_{\mathrm{F}}$, i.e., $n_{\mathrm{EF}-5} \leq n \leq n_{\mathrm{EF}+5}$. (3) Considering the requirement of broken time reversal symmetry, the spin components for each pair should be in opposite directions on the $x y$ plane, i.e., $S_{x, y}\left(\mathbf{k}_{1} /\right.$ $\left.\mathbf{k}_{3}, n\right)=-S_{x, y}\left(\mathbf{k}_{2} / \mathbf{k}_{4}, n\right)$; meanwhile, $S_{z}\left(\mathbf{k}_{i}, n\right)=0, i=1,2,3$, 4. (4) The $\mathbf{k}$ points on the same side of $\mathbf{k}_{c}$ should possess the same directions of spin on the $x y$ plane, i.e., $S_{x, y}\left(\mathbf{k}_{1} / \mathbf{k}_{2}, n\right) \cdot S_{x, y}\left(\mathbf{k}_{3} / \mathbf{k}_{4}, n\right)>0$.
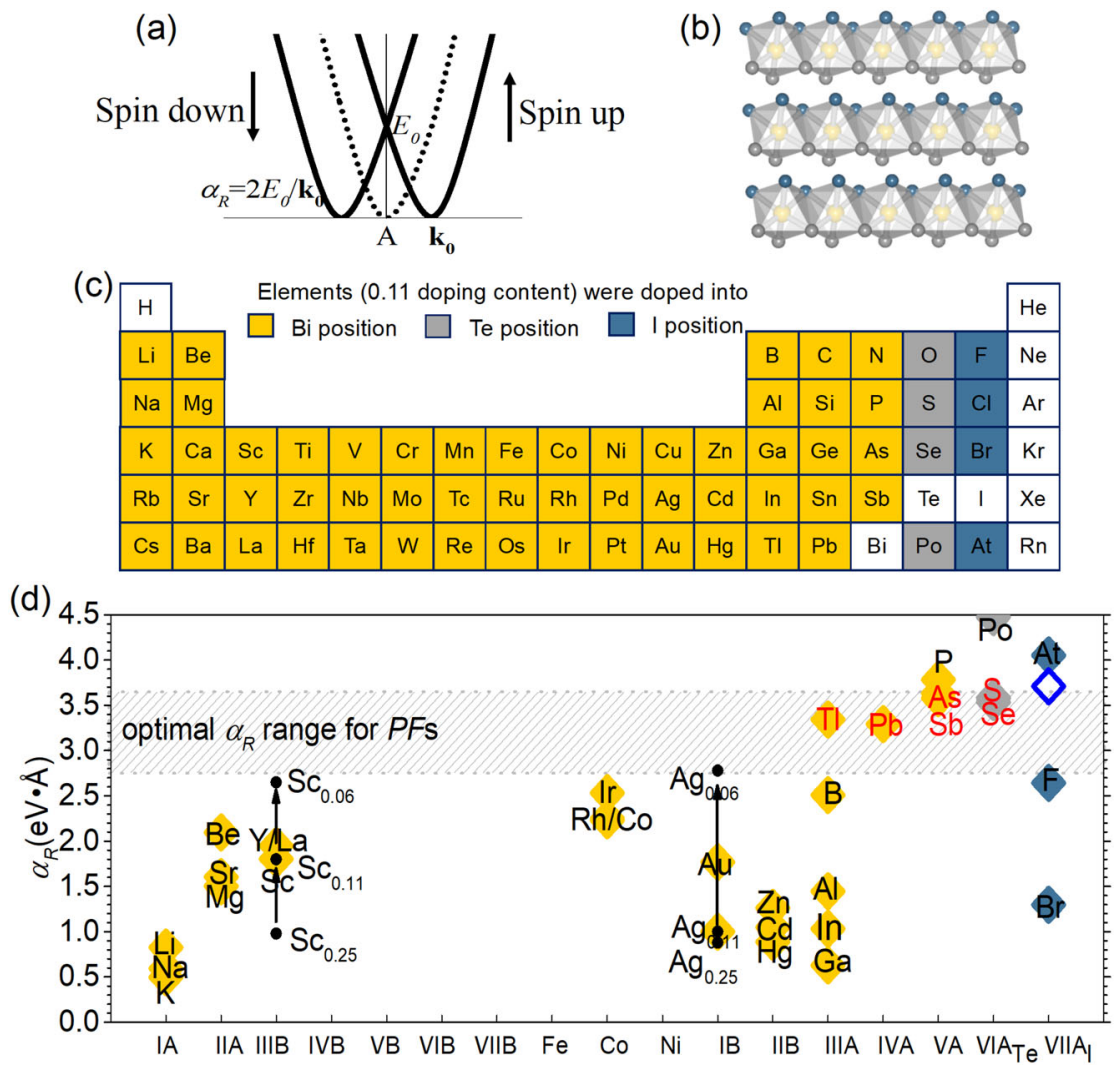

Fig. 1 Tunable $\boldsymbol{a}_{\mathrm{R}}$ in doped BiTel. a Schematic illustration of the Rashba band structure near the conduction band minimum of BiTel. b Crystal structure of BiTel. c Dopants considered in this study. d Dopant $-a_{R}$ map for BiTel with a doping content of 0.11 . The effect of doping content variation on $a_{\mathrm{R}}$ is also calculated for the two dopants Sc and Ag. The colors of the elements marked in $\mathbf{d}$ correspond to the colors in c. The hollow diamond indicates the $a_{R}$ of pristine BiTel $\left(a_{R}=3.71 \mathrm{eV} \AA\right)$. The gray rectangle in d shows the optimal $a_{R}$ range for thermoelectric PFs. 

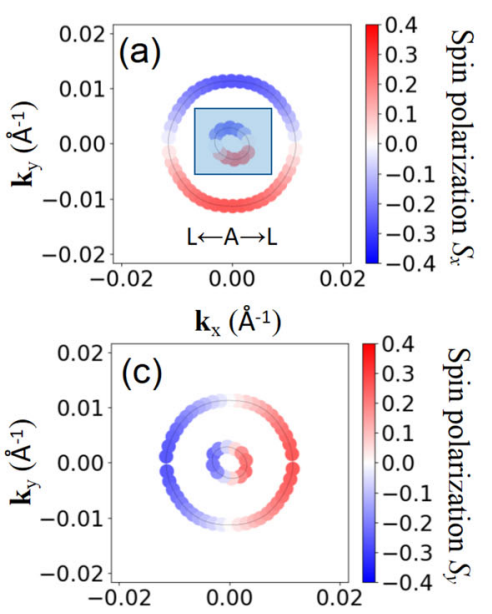

$\mathbf{k}_{\mathrm{x}}\left(\AA^{-1}\right)$ (b)
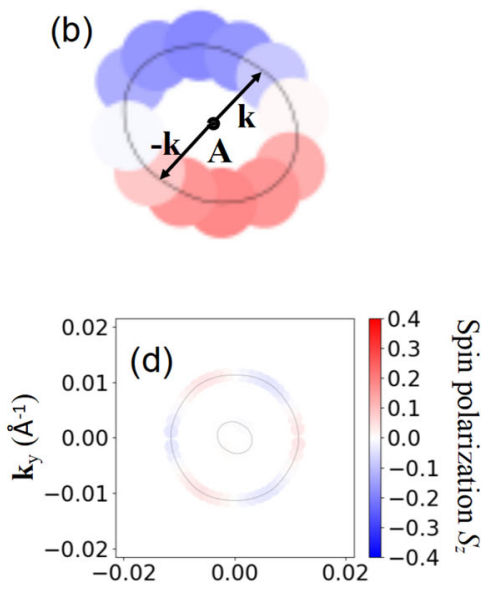

$\mathbf{k}_{\mathrm{x}}\left(\AA^{-1}\right)$

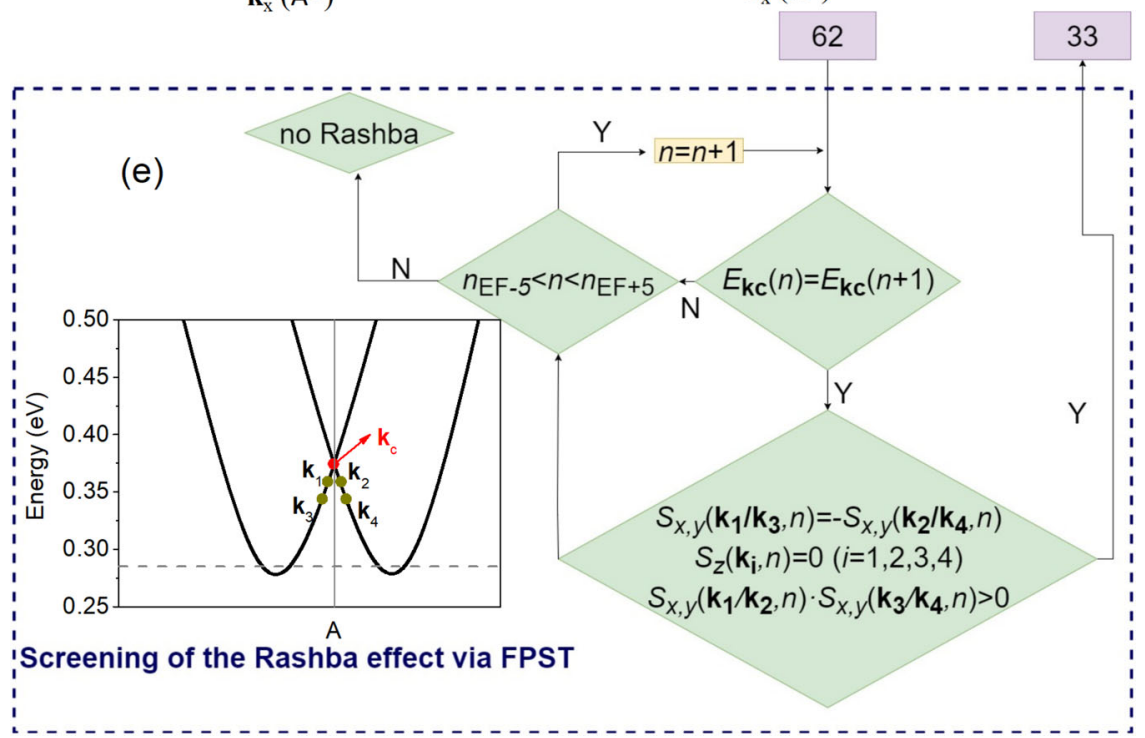

Fig. 2 High-throughput screening of doped BiTel with the Rashba effect. a-d Two-dimensional Fermi surface spin textures of BiTel with Rashba splitting at the energy level labeled by the gray dashed line in the inset of e. The colors indicate the magnitude of the spin polarization along the (a) $x,(\mathbf{c}) y$, and (d) $z$ directions. b Enlarged figure of the shaded part of a. e Workflow of the FPST method for HTP screening of the Rashba effect. The five points are labeled in the inset of e. See the text for details.

Systems with any of the above requirements violated are tagged as non-Rashba systems. This is the main workflow of the FPST method, and allows the identification of systems that have significant Rashba spin splitting. Since the FPST method only needs the information for five $\mathbf{k}$ points, it can accelerate the identification of Rashba-split bands and avoid the high computational cost of direct calculation of the whole complex band structures with SOC. Thus, it provides a simple screening process that is amenable to HTP searching. The method is applicable not only for screening the doped BiTel but also for other materials that potentially have the Rashba effect. In order to further lower the computational cost, an alternative three-point-spin-texture method is also proposed, as shown in Supplementary Fig. 2, which only requires the information for $\mathbf{k}_{c}, \mathbf{k}_{1}$, and $\mathbf{k}_{2}$.

Three dominant factors for tuning Rashba parameters We now turn to the results for doped BiTel. The FPST method is used to screen 33 dopants in BiTel. The band structures of these doped BiTel materials are calculated. Suitable semiconducting band structures are obtained for 32 of them. The band structures are shown in Supplementary Fig. 3. The values of $a_{\mathrm{R}}$ extracted from these are shown in the $a_{R}$-dopant map (Fig. $1 \mathrm{~d}$ ). Figure $1 \mathrm{~d}$ shows that the $a_{R} S$ of doped BiTel with dopants substituting on the $\mathrm{Bi}$ site are generally smaller than that of pristine BiTel. This is due to the relatively strong $\mathrm{SOC}$ strength of the $\mathrm{Bi}$ atom, which can be reflected by the SOC energy $E_{\mathrm{soc}}{ }^{25}$. In BiTel, the $E_{\mathrm{soc}}$ for the respective components are $1.91 \mathrm{eV}$ per electron for $\mathrm{Bi}, 0.39 \mathrm{eV}$ per electron for $\mathrm{Te}$, and $0.38 \mathrm{eV}$ per electron for $\mathrm{I}$. $\mathrm{Bi}$ atoms dominate the $E_{\text {soc }}$ of $\mathrm{BiTel}$. Therefore, the $\mathrm{Bi}$ concentration can roughly determine the SOC strength of the doped systems. With dopant atoms in groups IA, IIA, IIIA, IVA, VA, and transition groups, the $a_{\mathrm{R}} \mathrm{S}$ are less than that of pristine BiTel. This is because the dopants generally have much smaller $E_{\text {soc }}$ than $\mathrm{Bi}$ due to their smaller atomic numbers and because of the non-p-electron nature of the chemically important valence states. In Fig. $1 \mathrm{~d}$, the variation in $a_{\mathrm{R}}$ with doping content is illustrated using $\mathrm{Sc}$ and $\mathrm{Ag}$ as examples. The $a_{\mathrm{R}} \mathrm{S}$ are increased for lower contents of Sc or Ag, which can also be understood by the correspondingly larger $\mathrm{Bi}$ content.

However, the underlying physics is more complicated than simply the effect of changing $\mathrm{Bi}$ content and thereby changing effective strength of the SOC; notably, the $a_{R} s$ of systems with some dopants, such as $\mathrm{P}, \mathrm{B}$, and Be with low atomic numbers, have surprisingly higher values than other systems with dopants in the same group. This is in contrast with the simple idea that a 

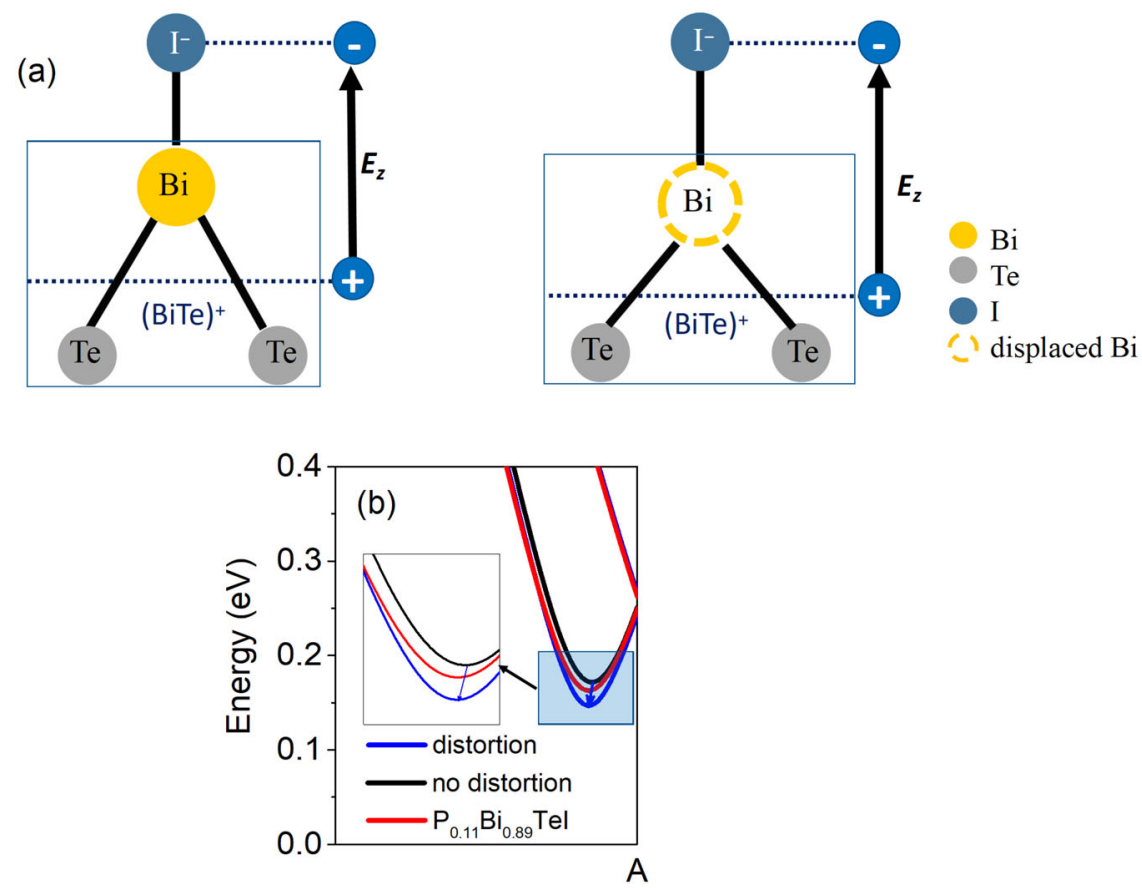

Fig. 3 The effect of local distortion on $\boldsymbol{a}_{\mathbf{R}}$. a Displacement of the crystal structure for BiTel with distortion similar to $\mathrm{P}_{0.11} \mathrm{Bi}_{0.89} \mathrm{Tel}_{\mathrm{B}} \mathbf{b}$ Band structures of BiTel with (blue line) and without (black line) distortion and $\mathrm{P}_{0.11} \mathrm{Bi}_{0.89} \mathrm{Tel}$ (red line).

higher atomic number should lead to larger Rashba splitting. Therefore, there must be another factor that can affect the Rashba strength in addition to the strength of the atomic SOC. We find that these small dopants move towards the Te atoms in BiTel. This generates local strains inside the crystallographic cell, which in turn would be expected to modify the strength of the effect of centrosymmetry breaking on the electronic structure. These local strains increase the distance between the positively charged BiTe layer and the negatively charged I layer, thus increasing $E_{z}$ (Fig. $3 a)^{16}$. The dopant, $P$, is a useful example. In fully relaxed $P$-doped $\mathrm{BiTel}$, the $\mathrm{P}$ atom is closer to the Te atoms, forming a much shorter $\mathrm{P}-\mathrm{Te}$ bond of $2.61 \AA$; in comparison, the $\mathrm{Bi}-\mathrm{Te}$ bond in pristine $\mathrm{BiTel}$ is $3.01 \AA$. Due to the existence of the local strain, $\mathrm{P}_{0.11} \mathrm{Bi}_{0.89} \mathrm{Tel}$ has an $a_{\mathrm{R}}(3.79 \mathrm{eV} \AA)$ comparable to that of pristine BiTel $(3.71 \mathrm{eV}$ $\AA)$. This indicates that the local strain compensates for the small $E_{\text {soc }}$ of $\mathrm{P}(0.00025 \mathrm{eV}$ per electron), as shown in Fig. $3 \mathrm{~b}$. To test this, we construct a distorted BiTel structure in Fig. $3 \mathrm{~b}$, with the same atomic positions as for the P-doped BiTel. The dipole moment along the $z$-axis can be estimated by $\mu_{z}=q \cdot d_{z}$, where $q$ is the charge of the positive BiTe/negative I layer, and $d_{z}$ is the distance between the layers (Fig. 3a). We find, by calculating the Bader charge ${ }^{26-28}$, that the $\mu_{z}$ s for distorted and undistorted BiTel are $2.08 \times 10^{-29}$ and $2.02 \times 10^{-29} \mathrm{C} \mathrm{m}$, respectively. In addition, there can be chemical changes in the bonding due to the different chemistries of $\mathrm{P}$ and $\mathrm{Bi}$. In any case, the increased dipole moment of distorted BiTel and contributions from chemical changes lead to an $a_{\mathrm{R}}$ as large as $4.31 \mathrm{eV} \AA$. Interestingly, the $a_{\mathrm{R}}$ of BiTel induced by another small dopant $\mathrm{Li}$ is $0.83 \mathrm{eV} \AA$, much smaller than that of $\mathrm{Be}$ $(2.10 \mathrm{eV} \AA)$. This is due to the fact that $\mathrm{Li}$ forms ionic bonds with the neighboring atoms, leading to no substantial local strain in the cell.

For the dopants in the VIA and VIIA groups that do not replace $\mathrm{Bi}, a_{\mathrm{R}}$ can also be tuned from 1.30 to $4.47 \mathrm{eV} \AA$. This is again a consequence of chemical effects and changes in the band gap of doped BiTel that affect the orbital character of the valence and conduction bands and further change the SOC energy splitting ${ }^{29}$. The SOC energy splitting mainly arises from the atomic spin-orbit contribution $^{30}$. The second-order perturbative correction in
Table 1. Variation in the band gap and $a_{\mathrm{R}}$ of systems with VIA and VIIA group dopants.

\begin{tabular}{lll}
\hline System & Band gap $(\mathrm{eV})$ & $a_{\mathrm{R}}(\mathrm{eV} \AA)$ \\
\hline BiTel & 0.35 & 3.71 \\
$\mathrm{BiTel}_{0.89} \mathrm{Br}_{0.11}$ & 0.85 & 1.30 \\
$\mathrm{BiTel}_{0.89} \mathrm{~F}_{0.11}$ & 0.41 & 2.64 \\
$\mathrm{BiTel}_{0.89} \mathrm{At}_{0.11}$ & 0.32 & 4.05 \\
$\mathrm{BiSe}_{0.11} \mathrm{Te}_{0.89} \mathrm{l}$ & 0.40 & 3.54 \\
$\mathrm{BiS}_{0.11} \mathrm{Te}_{0.89} \mathrm{l}$ & 0.43 & 3.58 \\
$\mathrm{BiPo}_{0.11} \mathrm{Te}_{0.89} \mathrm{l}$ & 0.25 & 4.47 \\
\hline
\end{tabular}

energy for this contribution is ${ }^{29}$

$\Delta \varepsilon_{m}^{(2)}(\mathbf{k})=\frac{\hbar}{m_{0}} \sum_{n \neq m} \frac{\left\langle u_{m}\left|H^{\mathrm{a}}\right| u_{n}\right\rangle\left\langle u_{n}|\mathbf{q} \cdot \mathbf{p}| u_{m}\right\rangle+\text { c.c. }}{\varepsilon_{m}-\varepsilon_{n}}$,

where $H^{\mathrm{a}}$ is the atomic contribution Hamiltonian term, c.c. denotes the complex conjugate, $u_{i}$ and $\varepsilon_{i}$ are the eigenstate and eigenenergy corresponding to state $i$ at $\mathbf{k}_{0}$, and $\mathbf{q}=\mathbf{k}-\mathbf{k}_{0}$. For BiTel, the same character of the conduction band and valence band leads to a large $\left\langle u_{\mathrm{CB}}\left|H^{\mathrm{a}}\right| u_{\mathrm{VB}}\right\rangle$ term. Here, $u_{\mathrm{CB}}$ and $u_{\mathrm{VB}}$ are for the conduction band and valence band, respectively. While in practice, the band structure has some polar character, with the valence bands having more Te character and the conduction bands having more Bi character, the qualitative expectation holds. In this way, a smaller $\varepsilon_{m}-\varepsilon_{n}$ is expected to be beneficial for a larger energy splitting, which further strengthens the Rashba effect. As shown in Table 1, most systems with smaller band gaps have larger $a_{\mathrm{R}} \mathrm{s}$.

Rashba engineering for optimizing power factors

As shown above, $a_{R}$ is tunable over a wide range. Therefore, it is of interest to explore the effects in applications where the performance is expected to depend on this parameter, particularly thermoelectrics. In thermoelectrics, it is known that the electrical 

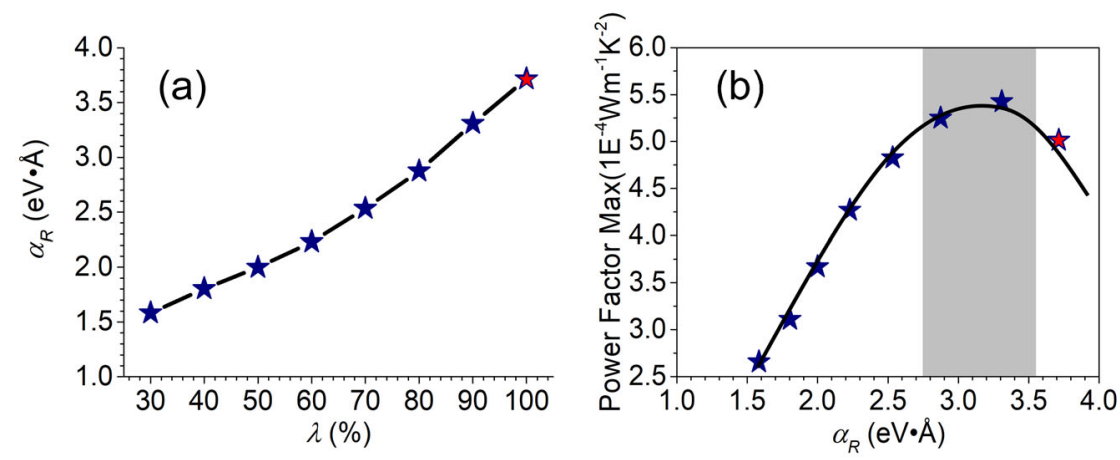

Fig. 4 The relationship between $\boldsymbol{a}_{\mathrm{R}}$ and PF. a Variation in $a_{\mathrm{R}}$ with the scaling factor of the SOC $\lambda$. $\mathbf{b}$ Relationship between $a_{\mathrm{R}}$ and the power factor maximum (PFM, maximum value of PF whose carrier concentration ranges from 0 to $10^{20} \mathrm{~cm}^{-3}$ ) at $300 \mathrm{~K}$.

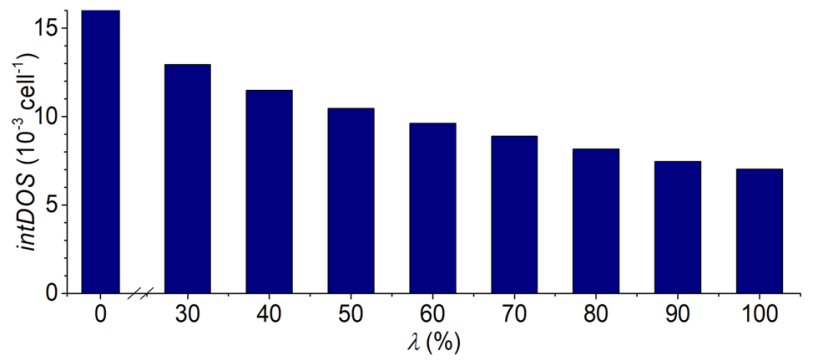

Fig. 5 The relationship between DOS and $\lambda$. Integrated DOS from the $\mathrm{CBM}$ to $\mathrm{CBM}+0.2 \mathrm{eV}$.

transport properties can be affected by changes in the electronic structure arising from the Rashba effect. We use the Transoptic package (http://mgi.shu.edu.cn/Portals/675/transoptic.zip) ${ }^{31}$ with the deformation potential method to solve the Boltzmann transport equation (details are given in the Supplementary

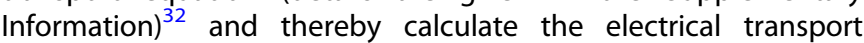
properties. The deformation potential of BiTel is $1.87 \mathrm{eV}$, and Young's modulus is $11.11 \mathrm{GPa}$. As shown in Supplementary Fig. 4, this approach yields results consistent with the experimental data ${ }^{5,33-35}$. All electrical transport properties presented here are calculated with the same deformation potential and Young's modulus, without the use of any undetermined parameter.

We start by exploring the relationship between $a_{R}$ and PF. We do this by changing the SOC strength, i.e., the scaling factor $\lambda$ in the SOC term ${ }^{36,37}$, and performing calculations for the pristine BiTel (we explore $30 \%, 40 \%, 50 \%, 60 \%, 70 \%, 80 \%, 90 \%$, and $100 \%$ of the physical $\lambda$ value). As $\lambda$ decreases, $a_{\mathrm{R}}$ correspondingly decreases due to the weaker SOC (Fig. 4a). Figure $4 \mathrm{~b}$ shows the variation in the power factor maximum at $300 \mathrm{~K}$ (PFM, maximum value of PF for the carrier concentration ranging from 0 to $10^{20} \mathrm{~cm}^{-3}$, which is the reasonable range for most thermoelectric materials) as a function of $a_{\mathrm{R}}$ (details are given in Supplementary Fig. 5). The optimal $a_{\mathrm{R}}$ range with a high PFM is highlighted by the gray rectangle. The red star represents $\lambda=100 \%$ with the value of $a_{R}=3.71 \mathrm{eV} \AA$, as in pristine BiTel. Figure $4 \mathrm{~b}$ shows that there is a higher PFM when the value of $a_{R}$ is $<3.71 \mathrm{eV} \AA$, i.e., $2.75 \mathrm{eV} \AA<a_{R}<3.55 \mathrm{eV} \AA$. With approximately equal $a_{R}$, Supplementary Fig. 6 proves that it is generally consistent between the electrical transport performances of pristine BiTel $\left(\lambda=40 \%\right.$ with $\left.a_{R}=1.80 \mathrm{eV} \AA\right)$ and doped BiTel $\left(\mathrm{Sc}_{0.11} \mathrm{Bi}_{0.89} \mathrm{Tel}\right.$ with $\left.a_{\mathrm{R}}=1.80 \mathrm{eV} \AA\right)$. It demonstrates that the $a_{\mathrm{R}}-\mathrm{PF}$ relationship for pristine BiTel is also suitable for doped BiTel. Based on the optimal $a_{\mathrm{R}}$ range of Fig. $4 \mathrm{~b}$, the ideal doping elements for thermoelectrics can be screened, which are highlighted in red in the gray rectangle of Fig. $1 \mathrm{~d}$. The elements are $\mathrm{Tl}, \mathrm{Pb}, \mathrm{As}, \mathrm{Sb}, \mathrm{S}$, and $\mathrm{Se}$, all of which are from the $p$-electron area of the periodic table.
The results in Fig. 4b offer another strategy of optimizing the power factors of thermoelectric materials, in addition to band engineering and scattering engineering ${ }^{24}$. By explicitly tuning the Rashba parameter, the electronic structures of materials can be largely regulated due to "defect-mediated Rashba engineering". Furthermore, the modifications mentioned in this work can be achieved by regular dopants in BiTel, which is convenient for thermoelectric experiments.

To obtain a physical understanding of Fig. $4 \mathrm{~b}$, the variation in the DOS around the CBM is analyzed. Figure 5 shows the integration of the DOS from the CBM to $C B M+0.2 \mathrm{eV}$ as a function of $\lambda$. When $\lambda$ decreases, the DOS correspondingly increases. This is due to the split band arising from the Rashba effect changing the effective mass of the band edge (details can be seen in Supplementary Fig. 7). High thermopower materials generally have large effective masses, which might detrimentally enhance the carrier scattering phase space and resistivity. The increased CBM DOS due to decreased $\lambda$ has beneficial effects for both the thermopower and resistivity. Therefore, there exists an optimal $a_{R}$ range for power factors, which is $2.75 \mathrm{eV} \AA<a_{R}<$ $3.55 \mathrm{eV} \AA$ in BiTel, as shown in Figs $1 \mathrm{~d}$ and $4 \mathrm{~b}$. This is a consequence of the detailed band shapes and illustrates the importance of the band structure for the thermoelectric performance.

In conclusion, a HTP method, FPST, is developed and applied to find dopants that lead to different strengths of the Rashba splittings in BiTel. We find that very strong modifications can be obtained, implying strong tunability via chemical doping. The variation in $a_{\mathrm{R}}$ is influenced by the strength of the SOC, band gap, and local strain. These different Rashba strengths modulate the electrical transport properties. Importantly, we find that there is an optimal range for $a_{R}$, and it is possible to tune it into this optimal range by doping. $a_{\mathrm{R}}$ can therefore serve as a descriptor for doped $\mathrm{n}$-type BiTel thermoelectrics. We find that the $a_{\mathrm{R}} \mathrm{s}$ of six particular doped compositions $\left(\mathrm{Tl}_{0.89} \mathrm{Bi}_{0.11}\right.$ Tel, $\mathrm{Pb}_{0.89} \mathrm{Bi}_{0.11} \mathrm{Tel}, \mathrm{As}_{0.11} \mathrm{Bi}_{0.89} \mathrm{Tel}$, $\mathrm{Sb}_{0.11} \mathrm{Bi}_{0.89} \mathrm{Tel}, \mathrm{BiSe}_{0.11} \mathrm{Te}_{0.89} \mathrm{l}$, and $\left.\mathrm{BiS}_{0.11} \mathrm{Te}_{0.89} \mathrm{l}\right)$ are likely to be in the optimal range of $2.75-3.55 \mathrm{eV} \AA$ for favorable electrical transport properties. Our calculations thus indicate the possibility of optimizing the electrical transport properties of thermoelectric materials through defect-mediated Rashba engineering. This is in particular enabled by varying the dopants and their concentrations. We also note that the strong modulation of the Rashba parameter by doping, as found here, could also play an effective role in engineering other functional materials.

\section{METHODS}

Basics of pristine BiTel and doping preference

The crystal structure of BiTel consists of alternating $\mathrm{Bi}, \mathrm{Te}$, and I layers (Fig. 1b). Bader charge analysis ${ }^{26-28}$ of BiTel shows that the charge state is +0.89 for the $\mathrm{Bi}$ atom, -0.42 for the Te atom, and -0.47 for the I 
atom. Thus, the elements with stronger metallicity than Te and I are placed at the Bi site, which has a cation character. The VIA elements replace Te, while the VIIA elements replace I. All the doped BiTel systems are fully relaxed.

\section{Details of first-principles calculations}

All density functional theory calculations are performed using the Vienna ab initio Simulation Package ${ }^{38}$ with the projector augmented wave method ${ }^{39}$. The band structure and transport calculations are performed with the modified Becke-Johnson exchange potential ${ }^{40}$, which improves the accuracy of band gaps in semiconductors. The results are for fully relaxed crystal structures ${ }^{40,41}$. The SOC is taken into account in all calculations. The spin textures are drawn by PyProcar ${ }^{42}$.

\section{DATA AVAILABILITY}

The data that support the findings of this study are available from the corresponding author upon reasonable request.

Received: 16 April 2020; Accepted: 2 July 2020;

Published online: 28 July 2020

\section{REFERENCES}

1. Di, S. D., Barone, P., Bertacco, R. \& Picozzi, S. Electric control of the giant Rashba effect in bulk GeTe. Adv. Mater. 25, 509-513 (2013).

2. Yang, J., Lei, C., Rong, C. \& Jiang, K. Conductance properties in spin field-effect transistors. Eur. Phys. J. B 62, 263-266 (2008)

3. Liu, G., Zhang, P., Wang, Z. \& Li, S.-S. Spin Hall effect on the kagome lattice with Rashba spin-orbit interaction. Phys. Rev. B 79, 035323 (2009).

4. Mirhosseini, $\mathrm{H}$. et al. Unconventional spin topology in surface alloys with Rashbatype spin splitting. Phys. Rev. B 79, 245428 (2009).

5. Ideue, T. et al. Thermoelectric probe for Fermi surface topology in the threedimensional Rashba semiconductor BiTel. Phys. Rev. B 92, 115144 (2015).

6. Cappelluti, E., Grimaldi, C. \& Marsiglio, F. Topological change of the Fermi surface in low-density Rashba gases: application to superconductivity. Physi. Rev. Lett. 98, 167002 (2007).

7. Mathias, S. et al. Quantum-well-induced giant spin-orbit splitting. Phys. Rev. Lett. 104, 066802 (2010).

8. Bychkov, Y. A. \& Rashba, É. I. Properties of a 2D electron gas with lifted spectral degeneracy. JETP lett. 39, 78 (1984).

9. $\mathrm{Wu}$, L. et al. Two-dimensional thermoelectrics with Rashba spin-split bands in bulk BiTel. Phys. Rev. B 90, 195210 (2014).

10. Caviglia, A. et al. Tunable Rashba spin-orbit interaction at oxide interfaces. Phys. Rev. Lett. 104, 126803 (2010).

11. Zhang, S.-j, Ji, W.-x, Zhang, C.-w, Li, P. \& Wang, P.-j Two-dimensional large gap topological insulators with tunable Rashba spin-orbit coupling in group-IV films. Sci. Rep. 7, 45923 (2017).

12. Liu, Q., Guo, Y. \& Freeman, A. J. Tunable Rashba effect in two-dimensional LaOBiS2 films: ultrathin candidates for spin field effect transistors. Nano Lett. 13, 5264-5270 (2013)

13. Grundler, D. Large Rashba splitting in InAs quantum wells due to electron wave function penetration into the barrier layers. Phys. Rev. Lett. 84, 6074 (2000).

14. Manchon, A., Koo, H. C., Nitta, J., Frolov, S. \& Duine, R. New perspectives for Rashba spin-orbit coupling. Nat. Mater. 14, 871-882 (2015).

15. Maaß, H. et al. Spin-texture inversion in the giant Rashba semiconductor BiTel. Nat. Commun. 7, 11621 (2016).

16. Ishizaka, K. et al. Giant Rashba-type spin splitting in bulk BiTel. Nat. Mater. 10, 521-526 (2011).

17. Eremeev, S. V., Nechaev, I. A., Koroteev, Y. M., Echenique, P. M. \& Chulkov, E. V. Ideal two-dimensional electron systems with a giant Rashba-type spin splitting in real materials: surfaces of bismuth tellurohalides. Phys. Rev. Lett. 108, 246802-246602 (2012).

18. Sakano, M. et al. Strongly spin-orbit coupled two-dimensional electron gas emerging near the surface of polar semiconductors. Phys. Rev. Lett. 110, 107204 (2012).

19. Liu, Q., Zhang, X., Luo, J. W., Freeman, A. \& Zunger, A. in Aps March Meeting (2014).

20. Stroppa, A. et al. Tunable ferroelectric polarization and its interplay with spin-orbit coupling in tin iodide perovskites. Nat. Commun. 5, 5900 (2014).
21. Kim, M., Im, J., Freeman, A. J., Ihm, J. \& Jin, H. Switchable $S=1 / 2$ and $J=1 / 2$ Rashba bands in ferroelectric halide perovskites. Proc. Natl Acad. Sci. USA 111, 6900-6904 (2014).

22. King, P. et al. Large tunable Rashba spin splitting of a two-dimensional electron gas in $\mathrm{Bi}_{2} \mathrm{Se}_{3}$. Phys. Rev. Lett. 107, 096802 (2011).

23. $\mathrm{Wu}$, L. et al. Thermopower enhancement in quantum wells with the Rashba effect. Appl. Phys. Lett. 105, 202115 (2014).

24. Yang, J. et al. On the tuning of electrical and thermal transport in thermoelectrics: an integrated theory-experiment perspective. NPJ Comput. Mater. 2, 15015 (2016).

25. Steiner, S., Khmelevskyi, S., Marsmann, M. \& Kresse, G. Calculation of the magnetic anisotropy with projected-augmented-wave methodology and the case study of disordered Fe1-xCox alloys. Phys. Rev. B 93, 224425 (2016).

26. Henkelman, G., Arnaldsson, A. \& Jónsson, H. A fast and robust algorithm for Bader decomposition of charge density. Comput. Mater. Sci. 36, 354-360 (2006).

27. Edward, S., Kenny, S. D., Roger, S. \& Graeme, H. Improved grid-based algorithm for Bader charge allocation. J. Comput. Chem. 28, 899-908 (2010).

28. Tang, W., Sanville, E. \& Henkelman, G. A grid-based Bader analysis algorithm without lattice bias. J. Phys.: Condens. Matter 21, 084204 (2009).

29. Bahramy, M. S., Arita, R. \& Nagaosa, N. Origin of giant bulk Rashba splitting: application to BiTel. Phys. Rev. B 84, 041202 (2011).

30. Kane, E. O. Energy band structure in p-type germanium and silicon. J. Phys. Chem. Solids 1, 82-99 (1956).

31. Yang, J., Xi, L., Zhang, W., Chen, L. \& Yang, J. Electrical transport properties of filled CoSb 3 skutterudites: a theoretical study. J. Electron. Mater. 38, 1397-1401 (2009)

32. $\mathrm{Xi}$, L. et al. Discovery of high-performance thermoelectric chalcogenides through reliable high-throughput material screening. J. Am. Chem. Soc. 140, 10785-10793 (2018).

33. $\mathrm{Wu}, \mathrm{L}$. et al. Enhanced thermoelectric performance in the Rashba semiconductor BiTel through band gap engineering. J. Phys.: Condens. Matter 28, 085801 (2016).Welcome@1234

34. Horák, J., Tichý, L. \& Lošták, P. Electrical conductivity of BiTel crystals. Phys. Status Solidi 63, 407-416 (2010).

35. Kulbachinskii, V., Kytin, V., Kudryashov, A. \& Shevelkov, A. in AIP Conference Proceedings, 358-361 (American Institute of Physics, 2012).

36. Kiss, A., Szolnoki, L. \& Simon, F. The Elliott-Yafet theory of spin relaxation generalized for large spin-orbit coupling. Sci. Rep. 6, 22706 (2016).

37. Wang, Y.-p, Ji, W.-x, Zhang, C.-w, Li, P. \& Wang, P.-j A new topological crystalline insulator in two-dimensional PbPo with tunable large bulk gaps. J. Mater. Chem. C 4, 8745-8749 (2016).

38. Kresse, G. \& Furthmuller, J. Efficient iterative schemes for ab initio total-energy calculations using a plane-wave basis set. Phys. Rev. B 54, 11169-11186 (1996).

39. Blöchl, P. E. Projector augmented-wave method. Phys. Rev. B 50, 17953 (1994).

40. Tran, F. \& Blaha, P. Accurate band gaps of semiconductors and insulators with a semilocal exchange-correlation potential. Phys. Rev. Lett. 102, 226401 (2009).

41. Perdew, J. P. \& Wang, Y. Accurate and simple analytic representation of the electron-gas correlation energy. Phys. Rev. B 45, 13244 (1992).

42. Herath, U. et al. PyProcar: a Python library for electronic structure pre/postprocessing. Comput. Phys. Commun. 251, 107080 (2020).

\section{ACKNOWLEDGEMENTS}

This work was supported by the National Key Research and Development Program of China (Nos. 2017YFB0701600, 2018YFB0703600), the Natural Science Foundation of China (Grant Nos. 51632005, 11604200, 11674211, and 51761135127), and the 111 Project D16002. W.Z. also acknowledges the support from the Guangdong Innovation Research Team Project (No. 2017ZT07C062), Guangdong Provincial KeyLab program (No. 2019B030301001), Shenzhen Municipal Key-Lab program (ZDSYS20190902092905285), and Shenzhen Pengcheng-Scholarship Program. J.Y. acknowledges the financial support of the US National Science Foundation with award number 1915933.

\section{AUTHOR CONTRIBUTIONS}

The initial idea was developed by X.L. and Jiong Yang, and its implementation was discussed with L.W. and Jihui Yang. Y.S. performed the spin texture analysis. Jiong Yang, W.Z., S.H., and D.J.S. analyzed the results of the dopant- $a_{\mathrm{R}}$ map. All authors participated in the data analysis and writing and reading of the paper. Jiong Yang managed the project. 


\section{COMPETING INTERESTS}

The authors declare no competing interests.

\section{ADDITIONAL INFORMATION}

Supplementary information is available for this paper at https://doi.org/10.1038/ s41524-020-00378-4.

Correspondence and requests for materials should be addressed to J.Y. or W.Z.

Reprints and permission information is available at http://www.nature.com/ reprints

Publisher's note Springer Nature remains neutral with regard to jurisdictional claims in published maps and institutional affiliations.
Open Access This article is licensed under a Creative Commons Attribution 4.0 International License, which permits use, sharing, adaptation, distribution and reproduction in any medium or format, as long as you give appropriate credit to the original author(s) and the source, provide a link to the Creative Commons license, and indicate if changes were made. The images or other third party material in this article are included in the article's Creative Commons license, unless indicated otherwise in a credit line to the material. If material is not included in the article's Creative Commons license and your intended use is not permitted by statutory regulation or exceeds the permitted use, you will need to obtain permission directly from the copyright holder. To view a copy of this license, visit http://creativecommons. org/licenses/by/4.0/.

(c) The Author(s) 2020 